MS31-04

\section{Influence of the hydrogen bond donors and acceptors ratio on the co-crystals formation features: the case of benzamide derivatives with dicarboxylic acids}

Aleksey Manin ${ }^{1}$, Ksenia Drozd ${ }^{1}$, Andrei Churakov ${ }^{2}$, German Perlovich ${ }^{1}$

1 Department of Physical Chemistry of drugs, G.A. Krestov Institute of Solution Chemistry of the Russian Academy of Sciences, Ivanovo, Russia

2. Department of Crystal Chemistry and X-ray Structure Analysis, Kurnakov Institute of General and Inorganic Chemistry, Russian Academy of Sciences, Moscow, Russia

email: alexnmanin@gmail.com

Co-crystals are multicomponent crystals that consist of two or more neutral molecules interacting with each other through non-covalent interactions. Co-crystals are well established in the literature as a route to new and alternative solid forms, and a promising method for fine-tuning properties of a molecule. The design of new co-crystals requires an understanding of hydrogen bonding functional groups and the supramolecular synthons between the drug and the coformer. Synthons are sub-classified as homosynthons and heterosynthons, i.e. those between like and unlike functional groups, respectively. There are many design strategies for binary cocrystals as seen from the literature. A crystal engineering or supramolecular synthon-based approach is a convenient and common method used in the design of such systems, implementing frequently occurring motifs from the literature applicable to the functional groups present in the API of interest. [1]

Tothadi with coauthors published a series of papers devoted to the study of synthon modularity in binary and ternary co-crystals of benzamide derivatives with dicarboxylic acids. [2, 3] The authors suggested that dicarboxylic acid can be introduced between the molecules of the benzamide derivatives bound through the amide-amide homosynton to realize the packaging of the single-component diphenol crystal. Indeed, the theoretical strategy allowed the modularity of the synthons to be used to produce a series of co-crystals with 4-hydroxybenzamide in a stoichiometric ratio of $2: 1$. [2]

In our work, we continued the studies of Tothadi and tried to analyze how the additional donor of the hydrogen bond in the benzamide molecule will affect the packing of the co-crystals with dicarboxylic acids. 4-aminobenzamide was chosen as the object of the study. It should be noted that the molecule of 4-aminobenzamide has not been studied well in the context of crystal engineering yet. Just four hits can be seen in the CSD corresponding to the pure compound and three cocrystals.

Seven new co-crystals and salts of 4-aminobenzamide with dicarboxylic acids and one new co-crystal of 4-hydroxybenzamide with malonic acid were obtained, monocrystals were grown and their crystal lattices were solved. DSC measurements were conducted to investigate the thermal behavior of BZA derivatives (4-AmBZA and 4-OHBZA) salt/cocrystals. The Xpac analysis was made for 34 known cocrystals of the p-derivatives of BZA. The similar "supramolecular constructs" of studied molecules crystal struc- tures were identified and the similarity of molecular packs was quantified.

This work was supported by the Russian Science Foundation (№ 17-73-10351).

References:

[1] Mapp, L.K. et al. (2017). Cryst. Growth Des. 17, 163-174

[2] Tothadi, S. \& Desiraju, G.R. (2012). Cryst. Growth Des. 12, 6188-6198;

[3] Tothadi, S. et al. (2014). Cryst. Growth Des. 14, 5293-5302

Keywords: aminobenzamide, intermolecular interactions 